\title{
A Modified Method for Hardness Determination from Nanoindentation Experiments with Imperfect Indenters
}

\author{
Wei Chao Guo, ${ }^{1}$ Heng Xu, ${ }^{2}$ Xin Qin Gao, ${ }^{1}$ Xiao Li Hou, ${ }^{1}$ and Yan Li ${ }^{1}$ \\ ${ }^{1}$ School of Mechanical and Instrumental Engineering, Xian University of Technology, Xian 710048, China \\ ${ }^{2}$ Business Intelligence and Knowledge Engineering Lab, Henan University of Technology, Zhengzhou 450001, China \\ Correspondence should be addressed to Wei Chao Guo; gwcletter@hotmail.com
}

Received 14 July 2015; Revised 2 November 2015; Accepted 30 November 2015

Academic Editor: Luciano Lamberti

Copyright (c) 2016 Wei Chao Guo et al. This is an open access article distributed under the Creative Commons Attribution License, which permits unrestricted use, distribution, and reproduction in any medium, provided the original work is properly cited.

\begin{abstract}
Nanoindentation is an effective nondestructive method for small scale determination of mechanical properties of materials. However, indentation response of metallic materials is very sensitive to indenter tip roundness, size effects, loading rate, and so forth. This study will analyze the effect of indenter shape imperfections on hardness determination. For this purpose, experimental investigations and finite element simulations are carried out. At first, it is found that hardness values determined with Oliver and Pharr's method are affected by errors caused by imperfect indenter tip: errors increase for imperfect indenters with larger tip radii. Afterwards, several commonly used methods accounting at different extents for tip radius variations are compared. However, most of those methods are found not to be accurate for shallow indentation. For this reason, a novel hardness determination method based on geometrical relations of the imperfect indenter tip is developed. Results show that the new approach is very effective even in the case of shallow indentation.
\end{abstract}

\section{Introduction}

The mechanical characterization of materials has long been represented by their hardness values. In order to correctly estimate the hardness, depth-sensing nanoindentation testing has been widely used for many years. Unlike other methods, nanoindentation equipment can continuously record the load $P$ versus the penetration depth $h$ curve. Fitting algorithms are used to process the force-indentation curve in order to determine the hardness and Young's modulus of the indented materials immediately. It is possible to carry out such test at microstructural scales (even at micro- and nanoscales), which makes this technique one of the most powerful tools for characterizing bulk and thin film materials. Experimental investigations of nanoindentation have been conducted on many materials to extract the hardness and other mechanical properties such as Young's modulus and residual stress $[1$, 2]. At present, advanced nanoindentation equipment can provide accurate measurements of the continuous variation of indentation load $P$ down to $\mu \mathrm{N}$, as a function of the indentation depth $h$ down to $\mathrm{nm}$.
However, in spite of those advantages, it is never possible that an indenter has a perfect shape as designed in nanoindentation measurements. It remains difficult to have the tip perfectly manufactured with no dissymmetry or defects, even with the best available grinding and polishing techniques. According to the report of MTS (http://www.mtsnano.com/ products/), the radius of a new indenter tip is in the range of $40 \mathrm{~nm}-50 \mathrm{~nm}$. However, indenter is usually worn soon in daily measurement. The radius of an indenter tip is generally considered as $50 \mathrm{~nm}-100 \mathrm{~nm}$ for a new indenter and $200 \mathrm{~nm}-$ $400 \mathrm{~nm}$ for used indenters $[3,4]$.

As nanoindentation tests are usually performed at the $\mathrm{micro/nanoscales,} \mathrm{even} \mathrm{small} \mathrm{defects} \mathrm{in} \mathrm{the} \mathrm{indenter} \mathrm{tip} \mathrm{may}$ lead to large differences in measurement results or even wrong results. Therefore, Oliver and Pharr [5] suggested that the contact area produced by an indenter should be calibrated by utilizing known materials before doing an indentation test. However, calibration process is often bypassed as it is not simple. It can be seen from a large number of publications [69] that material properties are evaluated through the indenters, which are usually assumed to have a perfect geometrical shape. In recent years, the effect of the indenter tips on 
measurement results has been deeply understood through theoretical models and experimental investigations [10-13]. Some researchers demonstrated that uncertainties caused by deviations from the indenter's nominal shape may be very significant. Particularly at micro- or nanoscales (indentation depth $h<0.2 \mu \mathrm{m}$ ), shape imperfections are affected by a large extent the level of uncertainty in the hardness measurements [10]. Furthermore, investigations have been performed in order to relate the indenter's shape imperfections with the material properties to be determined. For example, Antunes et al. [13] estimated the effect of tip imperfections on the determination of Vickers hardness and Young's modulus. It was found that large differences were obtained with different sizes of tip imperfections. However, flat imperfect tips were considered in order to simplify the analysis. Bouzakis et al. [11] investigated the effects of the indenter tip geometry from Berkovich and Vickers tests, which were performed to calculate the stress-strain laws of materials. They pointed out that indenters of the same type, including different imperfections, lead to significantly different results.

The aim of this paper is to compare the existing methods for hardness determination utilizing nanoindentation and to seek a simple and effective method to minimize the effect of indenter tip imperfection.

\section{Methods for Hardness Determination}

Hardness is usually defined as the ability of a material to sustain deformation under the applied load. This deformation is derived from the classical theory for elastoplastic deformation. The nanoindentation machine continuously records the load $P$ versus the indentation depth $h$, during loading and unloading process; see Figure 1. According to [5, 6], for a sharp indentation of elastoplastic material, loading curvature is governed by both elastic and plastic properties of indented material. The response can be described by Kick's law (i.e., $P=C h^{2}$, where $C$ is a constant). However, unloading curve is mainly governed by elastic properties. The slope of the unloading $P$ - $h$ curve that is measured at the maximum load can describe the elastic modulus of indented material. The details can be seen in Section 2.1. Once $P$ - $h$ curve is recorded, the hardness can be defined as $[4,5]$

$$
H=\frac{P}{A_{\text {proj }}},
$$

where $A_{\text {proj }}$ is the projected contact area. As shown in Figure 2, if the material is tested with a conical indenter, the projected contact area, $A_{\text {proj }}$, can be calculated as

$$
A_{\text {proj }}=\pi a_{c}^{2}=\pi(h \tan \theta)^{2} .
$$

Here, $\theta$ is the semiangle of the conical indenter.

However, in real indentation experiments, it is not possible to have an indenter which has a perfect sharp tip. Figure 2 shows that the indenter usually has a round tip instead of a sharp tip. In indentation experiment, the real contact depth becomes $h_{c}$ instead of $h$, and the indentation depth will deviate by $h_{d}$ from the nominal value. Thus $A_{\text {proj }}$ will be

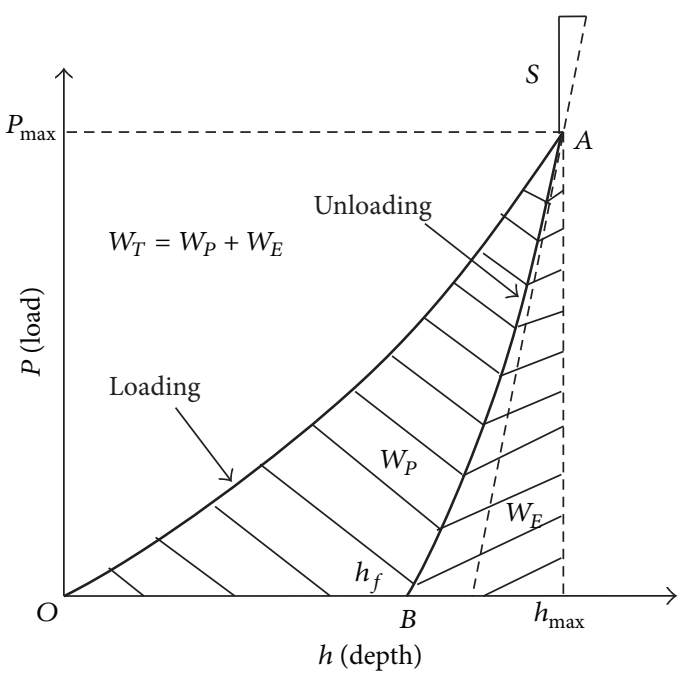

Figure 1: Typical indentation load-depth $(P-h)$ curve for elastoplastic material.

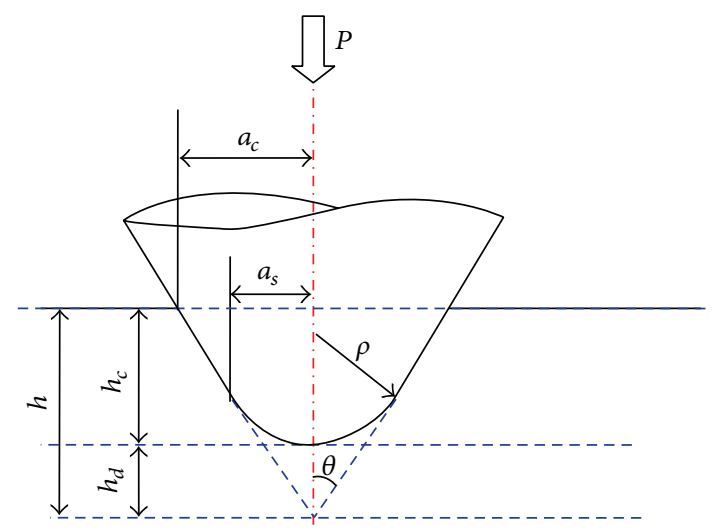

FIGURE 2: Schematic of the imperfect conical indentation profiles.

underestimated if $A_{\text {proj }}$ is still calculated by (2). Researchers proposed many methods to eliminate the error produced by the imperfect indenter tip, such as Oliver and Pharr, Thurn and Cook, and the work-of-indentation methods. All those methods will be compared in this study.

2.1. Oliver and Pharr's Method. The methodology proposed by Oliver and Pharr [5] is the most widely method used in current applications and nearly all the commercial depthsensing nanoindentation machines calculate hardness using this method. The hardness value can be inferred from the $P$ $h$ curve as follows:

$$
\begin{aligned}
H_{\mathrm{O} \& P} & =\frac{P}{A_{\text {proj }},} \\
S & =\left.\frac{d P}{d h}\right|_{P=P_{\max }},
\end{aligned}
$$




$$
\begin{aligned}
h_{c} & =h_{\max }-\varepsilon \frac{P_{\max }}{S}, \\
A_{\text {proj }} & =24.5 h_{c}^{2}+\sum_{i=1}^{8} C_{i} h_{c}^{1 / 2^{i-1}} .
\end{aligned}
$$

The actual profile of the indenter in contact with the tested material can be estimated with (4)-(6): $S$ is the "unloading stiffness" measured at the maximum penetration depth (see Figure 1); $\varepsilon$ is a geometrical constant and its value depends on the type of indenter (e.g., for a conical indenter, $\varepsilon=2(\pi-2) / \pi$; for a flat punch $\varepsilon=0.72) . C_{i}$ are the constants which describe the deviations due to an indenter tip. Those constants are calibrated by fitting the relations between $A_{\text {proj }}$ and $h_{c}$ of a known material. The process begins with the calculation of $h_{c}$ using (5) and the projected contact area $A_{\text {proj }}$ can be determined as

$$
A_{\text {proj }}=\frac{\pi S^{2}}{4 E_{r}^{2}},
$$

where $E_{r}$ is the reduced modulus defined as

$$
\frac{1}{E_{r}}=\frac{1-v^{2}}{E}+\frac{1-v_{i}^{2}}{E_{i}} .
$$

In (8), $E$ and $E_{i}$ are elastic moduli of the indented material and of the indenter; $v$ and $v_{i}$ are Poisson's ratios of the indented material and indenter, respectively. For a diamond indenter, $E_{i}=1140 \mathrm{GPa}$ and $v_{i}=0.07$. Since $E_{i}$ is nearly ten times larger than that of metals, the indenter can be considered a rigid body and the last part of (8) is assumed as zero. If we know the parameters of a known material, the reduced modulus $E_{r}$ can be obtained from (8). Subsequently, the constants $C_{i}$ in (6) can be calibrated from the experimental data gathered for this known material.

2.2. Thurn and Cook's Method. The drawback of Oliver and Pharr's method is the large number of parameters that must be calibrated. For this reason, Thurn and Cook [12] presented an improved method, which is related directly to tip-blunting or tip-rounding, to evaluate the projected contact area. They assumed that the profile of the indenter tip is the harmonic average of the conical tip and the spherical tip. The projected contact area is hence defined as

$$
A_{T \& C}=\pi\left(\frac{h_{c}}{\cot \theta}+2 \rho \cot \theta\right)^{2},
$$

where $\rho$ is the radius of curvature of the indenter tip.

2.3. Work-of-Indentation Method. Figure 1 shows that the total loading work $W_{T}$ done in the indentation process is comprised of two parts, $W_{P}$ and $W_{E}$, corresponding to plastic and elastic deformation, respectively. Therefore, it was suggested to determine hardness directly from the energy of indentation [3]. A strength point of this method is that hardness determination is marginally affected by indenter shape imperfections. The hardness, defined as the ratio between the maximum applied load and the projected contact area, is equivalent to the plastic work divided by the plastically deformed volume:

$$
H_{\text {Energy }}=\frac{P}{A_{\text {proj }}}=\frac{W_{P}}{V_{P}},
$$

where $V_{P}$ is the volume of plastic deformation. For the conical indenter nominal shape, $V_{P}$ can be determined as follows:

$$
V_{P}=\frac{1}{3} A_{\mathrm{proj}} h_{f}=\frac{1}{3} \pi h_{c}^{2} h_{f} \tan ^{2} \theta
$$

The plastic work $W_{P}$ can be determined as follows:

$$
W_{P}=W_{T}-W_{E}
$$

According to the schematic illustration of a $P$ - $h$ curve shown in Figure 1, the loading work $W_{T}$ done by the indenter in the loading phase is

$$
W_{T}=\int_{0}^{h_{\max }} P d h .
$$

It corresponds to the area under the loading curve $O A$. Similarly, the area underneath the unloading curve $A B$ is the total unloading work:

$$
W_{E}=-\int_{h_{\max }}^{h_{f}} P d h .
$$

$W_{E}$ also represents the reversible elastic work in the indentation process.

2.4. Modified Method. This study introduces a modified projected area function for an imperfect indenter. The method is derived from the geometrical relations of the imperfect indenter tip. As Figure 2 shows, it is assumed that an imperfect indenter normally includes a spherical tip and conical root as it is worn in frequent utilizations. Thus the contact area produced by an imperfect indenter normally includes two regions. At the beginning, the indenter just penetrates into the specimen at a shallow depth, and the contact area deforms only under the press of spherical tip. The contact area radius, $a_{s}$, can be written as

$$
a_{s}=\sqrt{\rho^{2}-\left(\rho-h_{c}\right)^{2}} \text {. }
$$

The modified projected contact area is calculated as

$$
A_{\text {Modified }}=\pi a_{s}^{2}=\pi\left[\rho^{2}-\left(\rho-h_{c}\right)^{2}\right] .
$$

Subsequently, the contact area is produced by both the spherical tip and the conical root when the indenter penetrates deeply. Therefore, the contact area is different from that produced by a perfectly conical indenter. However, the contact area can be calculated by redefining contact radius. According to Figure 2, the contact radius $a_{c}$ can be written as

$$
a_{c}=h \tan \theta=\left(h_{c}+h_{d}\right) \tan \theta ;
$$


here the deviation of penetration depth for the imperfect indenter $h_{d}$ can be expressed by

$$
h_{d}=\rho\left(\sin ^{-1} \theta-1\right) \text {. }
$$

By comparing (17) and (18), the modified projected contact area function for the imperfect indenter is expressed as

$$
A_{\text {Modified }}=\pi a_{c}^{2}=\pi\left(h_{c} \tan \theta+\frac{1-\sin \theta}{\cos \theta} \rho\right)^{2} .
$$

The hardness is hence determined with this modified method as

$$
H_{\text {Modified }}=\frac{P}{A_{\text {Modified }}} .
$$

It should be noted that the hardness can be easily determined as $\rho$ is the only parameter to be measured.

\section{Numerical Analysis of the Nanoindentation Process}

The effect of imperfect indenter tip on hardness determination is studied via numerical analysis because it is easy to set the tip radius in a large range of values in order to include almost worn indenter tips. Classical engineering metallic materials $[8,14]$ working in the elastoplastic regime are considered in this study (see Table 1). Materials considered in [8] were characterized with Vickers tests while the hardness of the material analyzed in [14] was determined using a conical indenter with semiangle of $70.3^{\circ}$ using Oliver and Pharr's method.

3.1. Modeling of Material Constitutive Behavior. For those elastoplastic models in $[8,14]$, Young's modulus is represented as $E$ and the initial yield stress is represented as $\sigma_{y}$. According to $[6,15]$, the true stress-strain $(\sigma-\varepsilon)$ curves of these materials can be closely approximated by the power law description:

$$
\sigma= \begin{cases}E \varepsilon, & \text { for } \sigma \leq \sigma_{y} \\ R \varepsilon^{n}, & \text { for } \sigma \geq \sigma_{y}\end{cases}
$$

where $E$ is Young's modulus, $\sigma_{y}$ is the initial yield stress, $n$ is the work-hardening exponent, and $R$ is the work-hardening rate expressed as

$$
R=\frac{E^{n}}{\sigma_{y}^{n-1}} .
$$

Because material plasticity is considered to be time independent, load history only contains the loading and unloading phases in the following studies.

Furthermore, in the following numerical calculations, Poisson's ratio is designated by $v$, and the von Mises plasticity with $J_{2}$ flow theory is assumed according to $[16,17]$. With the above assumptions and definitions, four independent parameters $\left(E, v, \sigma_{y}\right.$, and $\left.n\right)$ are required to completely
TABLE 1: Mechanical properties of the elastoplastic materials considered in this study.

\begin{tabular}{lcc}
\hline Name & $\left(E, v, \sigma_{y}, n\right)$ & $H(\mathrm{GPa})$ \\
\hline Stainless steel [8] & $(200 \mathrm{GPa}, 0.3,675 \mathrm{MPa}, 0.19)$ & 2.56 \\
Annealed copper [8] & $(110 \mathrm{GPa}, 0.32,20 \mathrm{MPa}, 0.52)$ & 0.5 \\
Aluminum alloy [14] & $(70 \mathrm{GPa}, 0.3,500 \mathrm{MPa}, 0.122)$ & 1.75 \\
\hline
\end{tabular}

characterize the elastoplastic properties of the indented materials (see Table 1). Concurrently, Coulomb's friction model is considered in the interfaces and the friction coefficient, $\mu=0.1$, is assumed in this paper. This modeling assumption is motivated by the fact that friction in the contact area should not be neglected. Previous studies $[6,8]$ indicate that the friction coefficient between well-polished metallic surfaces and diamond ranges between 0.1 and 0.15 .

3.2. Finite Element Model. Figure 3 shows the $2 \mathrm{D}$ axisymmetric finite element mode developed in ANSYS 14.0 to simulate the indentation responses of elastoplastic solids. The size of the specimen is $46 \mu \mathrm{m} \times 46 \mu \mathrm{m}$, which is filled by 15577 four-node quadrilateral elements (PLANE182). A finer mesh near the contact region and a gradually coarser mesh far from the contact region are designed to ensure numerical accuracy. The mesh is somehow irregular because the number of elements on the opposite edges of the model is not the same. Hence, the free meshing option available in ANSYS is selected. However, element size near the contact region is small enough to have accurate results. Convergence analysis is performed to prove that element skewness and aspect ratio are acceptable and lead to mesh-independent solutions even in the large deformation regime.

The left edge of the model is taken as axis of symmetry, which is constrained not to move in the horizontal direction. Similarly, bottom side nodes cannot move in the vertical direction. The semiangle of the conical indenter is $\theta=$ $70.3^{\circ}$, a typical value for the Berkovich and Vickers protocols. Furthermore, the tip radius of the imperfect conical indenter $\rho$ can vary in the range of $0-500 \mathrm{~nm}$. The contact problem is solved with the penalty method and the load is applied by controlling the indenter displacement. The maximum penetration depth $h_{\max }$ must not exceed $700 \mathrm{~nm}$. While the indenter reaches the maximum penetration depth, more than 350 nodes are in the contact region to guarantee the accuracy of finite element simulations.

\subsection{Estimation of Hardness for Different Imperfect Indenters.} Investigation of the effect of indenter tip actual shape on calculated hardness and evaluation of errors made in hardness determination are the core point of this study. Thus the projected contact area $A_{\text {proj }}$ is calculated only by the first part of (6) and the radius of the indenter tip is set to vary in the range of $0-500 \mathrm{~nm}$, which covers usually worn indenters. Load $P$ can be directly calculated in ANSYS. Values of hardness are computed with (1) and plotted in Figure 4. It can be seen that the hardness increases significantly with the tip radius. This is due to the fact that if an indenter has 


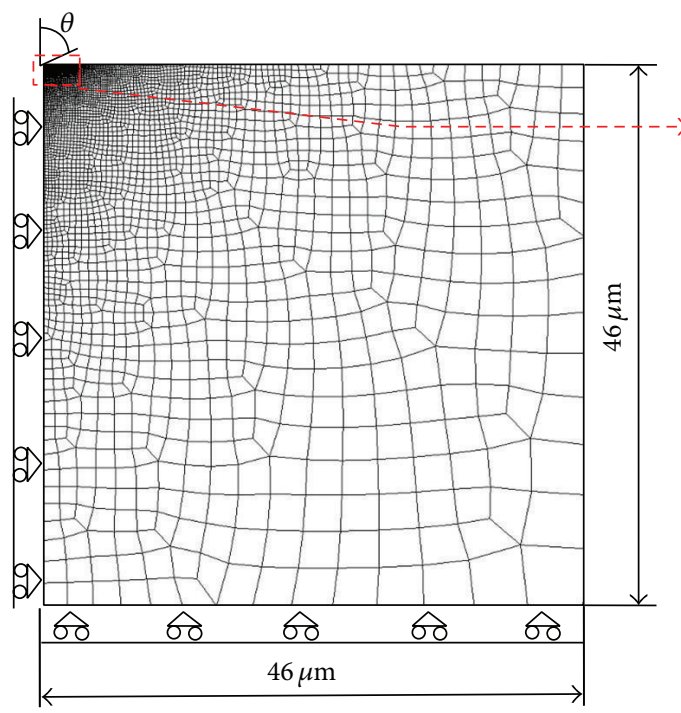

(a) Complete mesh

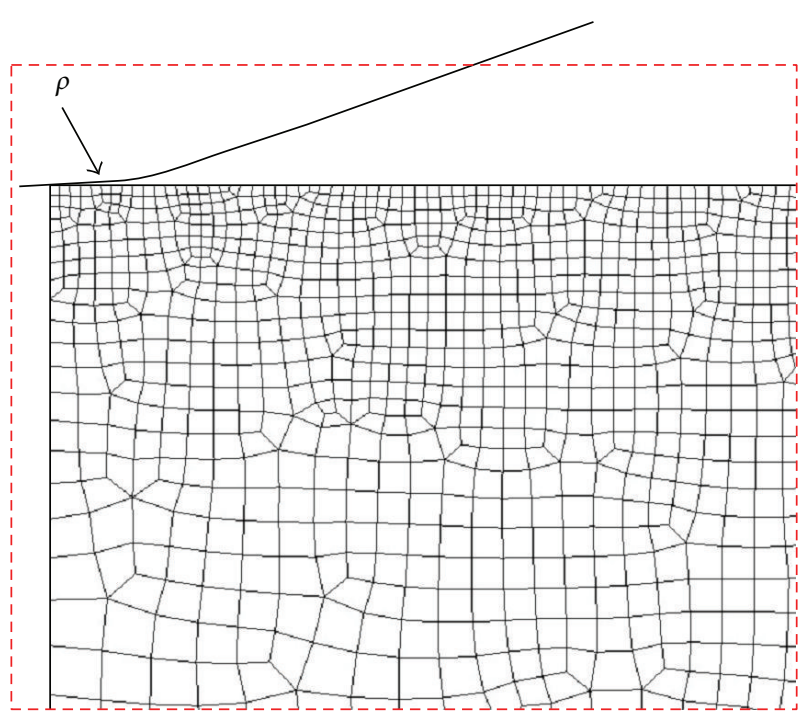

(b) Details of the contact region

FIGURE 3: Finite element model used in 2D numerical simulation.

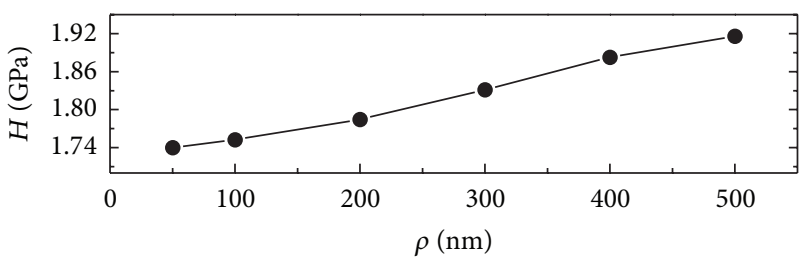

- - Aluminum alloy

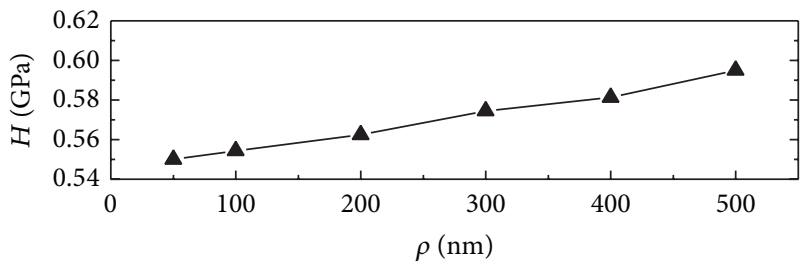

- - Annealed copper

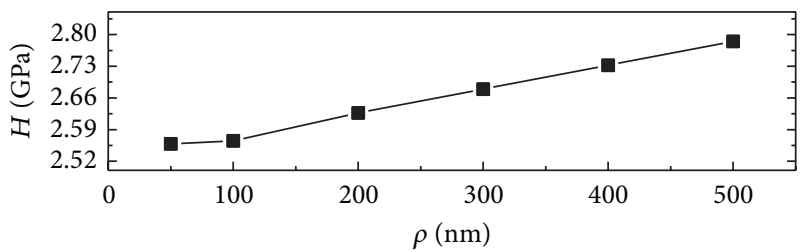

-匹- Stainless steel

Figure 4: The hardness estimated by imperfect indenters.

larger tip radius, the indentation depth will deviate more by $h_{d}$ from the nominal value (refer Figure 2). Hence, the contact area $A_{\text {proj }}$ will be underestimated more according to (2), and hardness will be overestimated more according to (1). For $\rho=500 \mathrm{~nm}$, the identified hardness equals 1.92, 0.60 , and $2.79 \mathrm{GPa}$, respectively, for aluminum alloy, annealed copper, and stainless steel. There is about $10 \%$ difference for each material compared with the referenced values listed in
Table 1. This confirms that the effect of actual indenter shape on hardness determination should not be neglected. It is therefore necessary to know the exact shape of the indenter in order to correct the errors in hardness determination.

\section{Comparison of Hardness Determination Methods}

Nanoindentation experiments on stainless steel, annealed copper, and aluminum alloy were carried out with the Nano Indenter G200 in the force loading mode. The conical indenter with semiangle of $70.3^{\circ}$ was not utilized in the experiments because most of the indenters available in our laboratory were seriously worn and included rounded tip radii greater than $900 \mathrm{~nm}$ measured by a field emission SEM, HITACHI S-4300, which has $1.5 \mathrm{~nm}$ resolution at acceleration voltage $15 \mathrm{kV}$. Furthermore, previous experiments carried out in our lab to compare the present data with the literature $[8,14]$, considered several types of indenters such as Vickers, Berkovich, and conical indenters with semiangles of $70.3^{\circ}$ and $60^{\circ}$. It was found that the measured hardness of the three investigated materials is less sensitive to the indenter shape than for other materials.

For this reason, the conical indenter with semiangle of $60^{\circ}$ and measured tip radius of $450 \mathrm{~nm}$ (also measured with HITACHI S-4300 SEM) was utilized. This allowed experiments to be carried out at the nanoscale. For each material, nine tests were carried out with different maximum penetration depth; that is, $h_{\max }$ varied from 112 to $1125 \mathrm{~nm}$. The $h_{\max } / \rho$ ratio hence varied between 0.25 and 2.5 , thus covering the limit cases of shallow indentation and deep indentation. Material hardness is also affected significantly by loading rate. According to $[6,18]$, determined hardness was affected significantly by loading rate, particularly in the case of fast loading process. In this paper, the indentation experiments were carried out under a relatively low loading 


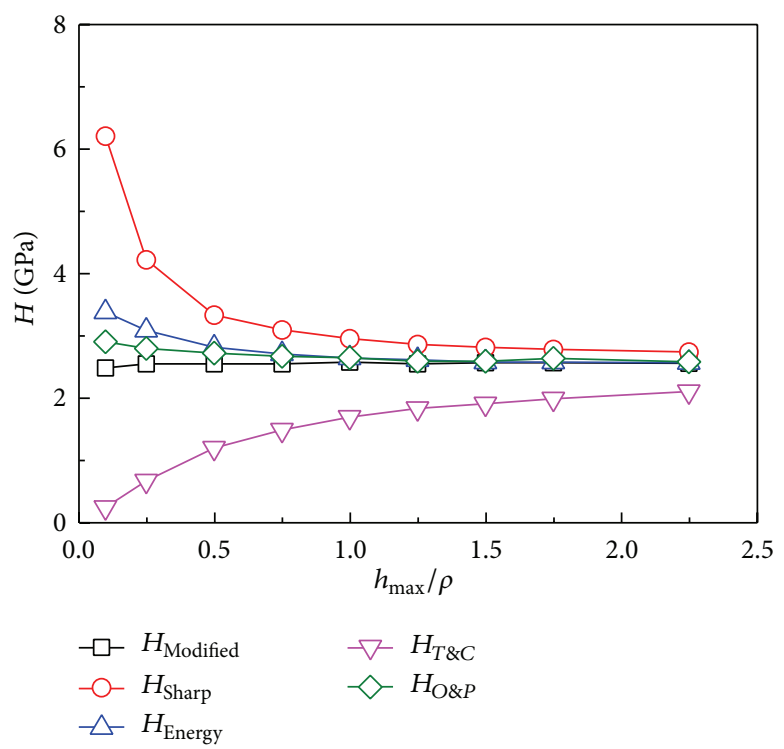

(a) Stainless steel

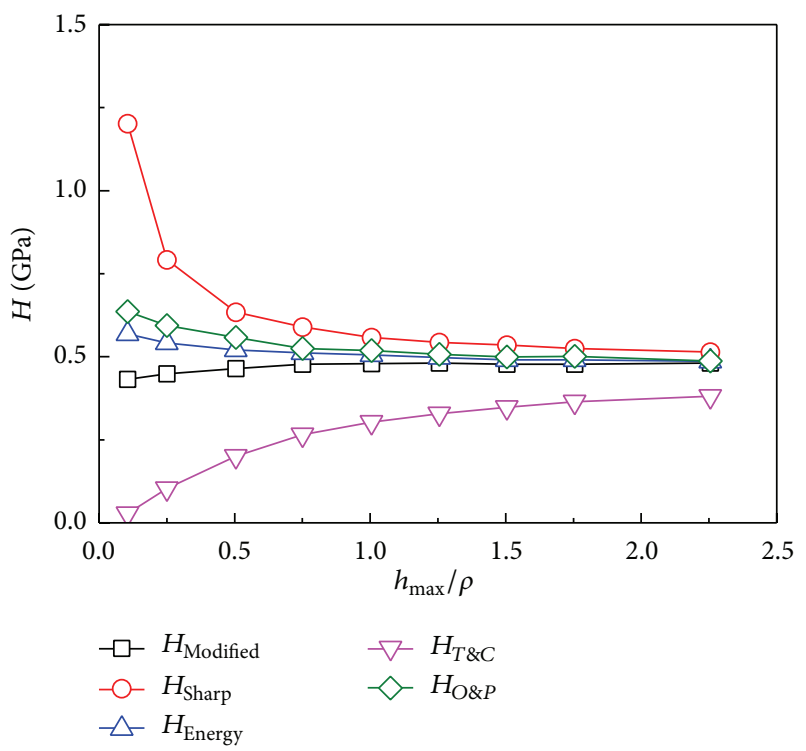

(b) Annealed copper

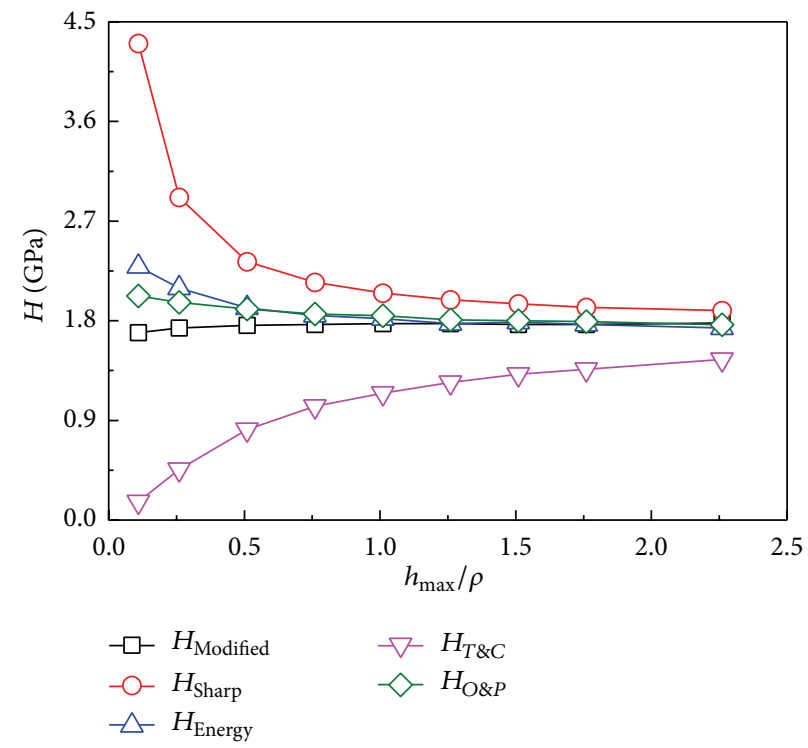

(c) Aluminum alloy

Figure 5: The hardness of materials determined by different methods.

rate to eliminate the effect of loading rate. Furthermore, in order to ensure that each experiment has nearly the same loading rate, loading/unloading times were set equal to 3 seconds for $h_{\max }=112 \mathrm{~nm}$ and 30 seconds for $h_{\max }=$ $1125 \mathrm{~nm}$.

Since it is hard to find indenters with large curvature radii as those considered in this study, the FE model developed to simulate nanoindentation tests is not directly validated against experimental results. However, the observed trend is in qualitative agreement with other experimental determinations. The values of hardness determined for the different indentation depths and methods are shown in Figure 5. The $x$-axis represents the ratio of $h_{\max } / \rho$, because the effect of indenter tip radius on hardness determination is usually a function of $h_{\max } / \rho$. Figure 5 shows that the hardness tends to reach the values listed in Table 1 with an increase of indentation depth. For the depth indentation, in particular it becomes constant for $h_{\max } / \rho=1.75$. This proves that the present trade study and the determined hardness are reliable. It can be concluded that the effect of indenter tip on hardness determination is negligible only in the deep indentation range or, at least, if the indentation depth is large enough compared to the tip radius.

In Figure $5, H_{\text {Sharp }}$ is the value of hardness determined without taking into account the effect of indenter tip; it is clearly seen that large errors occur for shallow indentations. That is because the projected contact area is in all likelihood produced only by rounded part of the indenter tip, similar to a spherical indenter. However, the projected contact area correction functions cannot exactly describe this scenario. 
The hardness values $H_{T \& C}$ determined with the Thurn and Cook method also show large errors for shallow indentation and, unlike other methods, increase with indentation depth. Furthermore, $H_{T \& C}$ values are always smaller than that determined by the other methods. This is due to the fact that the Thurn and Cook method overestimates projected contact area.

Hardness values determined with the present approach (the corresponding values of hardness are denoted as $H_{\text {Modified }}$ in Figure 5) are overall the most accurate. The present method is significantly less sensitive to penetration depth and does not vary much over the whole indentation range. This proved that the proposed method can effectively eliminate the influence of indenter tip in the process of hardness determination. The values of hardness $H_{O \& P}$ determined with the Oliver and Pharr method accounting for the effect of tip shape also show large errors at shallow indentation depth but tend to become constant already for $h_{\max } / \rho>0.25$. A similar behavior was observed for the work-of-indentation approach (the corresponding values of hardness are denoted as $H_{\text {Enegry }}$ in Figure 5) which is even more accurate than that by Oliver and Pharr method in the case of annealed copper.

\section{Conclusions}

This study analyzed in detail the effects of indenter geometric imperfections on the determination of hardness for metallic materials in the elastoplastic regime. A modified method was developed to eliminate errors made when experiments are carried out with imperfect indenters: the projected contact area was computed so as to account for the actual profile of the rounded indenter which deviates from the ideal conical shape. Results confirmed the validity of the proposed approach. While most of the methods available in literature were significantly affected by the penetration depth, hardness values determined with the proposed approach were practically constant regardless of the fact of being in shallow or depth indentation regime. The present method proved itself able to improve the accuracy of hardness determination even at extra shallow indentation depths.

\section{Conflict of Interests}

The authors declare that there is no conflict of interests regarding the publication of this paper.

\section{Acknowledgments}

The authors would like to gratefully acknowledge the funds from the National Natural Science Foundation of China (Grant nos. 51505377 and 71001034) and the Education Department of Shaanxi Province (Grant no. 102-00K1410) as well as the financial supports from University of Technology for young researchers (Grant no. 102-211408).

\section{References}

[1] L. Łatka, D. Chicot, A. Cattini, L. Pawłowski, and A. Ambroziak, "Modeling of elastic modulus and hardness determination by indentation of porous yttria stabilized zirconia coatings," Surface \& Coatings Technology, vol. 220, pp. 131-139, 2013.

[2] A. Karimzadeh, M. R. Ayatollahi, and M. Alizadeh, "Finite element simulation of nano-indentation experiment on aluminum 1100," Computational Materials Science, vol. 81, pp. 595-600, 2014.

[3] J. R. Tuck, A. M. Korsunskyl, S. J. Bull, and R. I. Davidson, "On the application of the work-of-indentation approach to depthsensing indentation experiments in coated systems," Surface \& Coatings Technology, vol. 137, no. 2-3, pp. 217-224, 2001.

[4] A. C. Fischer-Cripps, Nanoindentation, Springer, New York, NY, USA, 2002.

[5] W. C. Oliver and G. M. Pharr, "Improved technique for determining hardness and elastic modulus using load and displacement sensing indentation experiments," Journal of Materials Research, vol. 7, no. 6, pp. 1564-1583, 1992.

[6] M. Dao, N. Chollacoop, K. J. Van Vliet, T. A. Venkatesh, and S. Suresh, "Computational modeling of the forward and reverse problems in instrumented sharp indentation," Acta Materialia, vol. 49, no. 19, pp. 3899-3918, 2001.

[7] J. Luo and J. Lin, "A study on the determination of plastic properties of metals by instrumented indentation using two sharp indenters," International Journal of Solids and Structures, vol. 44, no. 18-19, pp. 5803-5817, 2007.

[8] M. Mata and J. Alcalá, "The role of friction on sharp indentation," Journal of the Mechanics and Physics of Solids, vol. 52, no. 1, pp. 145-165, 2004.

[9] Z. Tang, Y. Guo, Z. Jia, Y. Li, and Q. Wei, "Examining the effect of pileup on the accuracy of sharp indentation testing," Advances in Materials Science and Engineering, vol. 2015, Article ID 528729, 10 pages, 2015.

[10] J. Q. Zhang and M. Sakai, "Geometrical effect of pyramidal indenters on the elastoplastic contact behaviors of ceramics and metals," Materials Science and Engineering A: Structural Materials Properties Microstructure and Processing, vol. 381, no. 1-2, pp. 62-70, 2004.

[11] K.-D. Bouzakis, N. Michailidis, S. Hadjiyiannis, G. Skordaris, and G. Erkens, "The effect of specimen roughness and indenter tip geometry on the determination accuracy of thin hard coatings stress-strain laws by nanoindentation," Materials Characterization, vol. 49, no. 2, pp. 149-156, 2002.

[12] J. Thurn and R. F. Cook, "Simplified area function for sharp indenter tips in depth-sensing indentation," Journal of Materials Research, vol. 17, no. 5, pp. 1143-1146, 2002.

[13] J. M. Antunes, L. F. Menezes, and J. V. Fernandes, "Influence of Vickers tip imperfection on depth sensing indentation tests," International Journal of Solids and Structures, vol. 44, no. 9, pp. 2732-2747, 2007.

[14] J. L. Bucaille, S. Stauss, E. Felder, and J. Michler, “Determination of plastic properties of metals by instrumented indentation using different sharp indenters," Acta Materialia, vol. 51, no. 6, pp. 1663-1678, 2003.

[15] X. Chen, N. Ogasawara, M. Zhao, and N. Chiba, "On the uniqueness of measuring elastoplastic properties from indentation: the indistinguishable mystical materials," Journal of the Mechanics and Physics of Solids, vol. 55, no. 8, pp. 1618-1660, 2007.

[16] G. Rauchs, "Optimization-based material parameter identification in indentation testing for finite strain elasto-plasticity," ZAMM-Journal of Applied Mathematics and Mechanics, vol. 86, no. 7, pp. 539-562, 2006. 
[17] J. P. Ponthot, "Unified stress update algorithms for the numerical simulation of large deformation elasto-plastic and elastoviscoplastic processes," International Journal of Plasticity, vol. 18, no. 1, pp. 91-126, 2002.

[18] L. Chang and L. Zhang, "Mechanical behaviour characterisation of silicon and effect of loading rate on pop-in: a nanoindentation study under ultra-low loads," Materials Science and Engineering A, vol. 506, no. 1-2, pp. 125-129, 2009. 

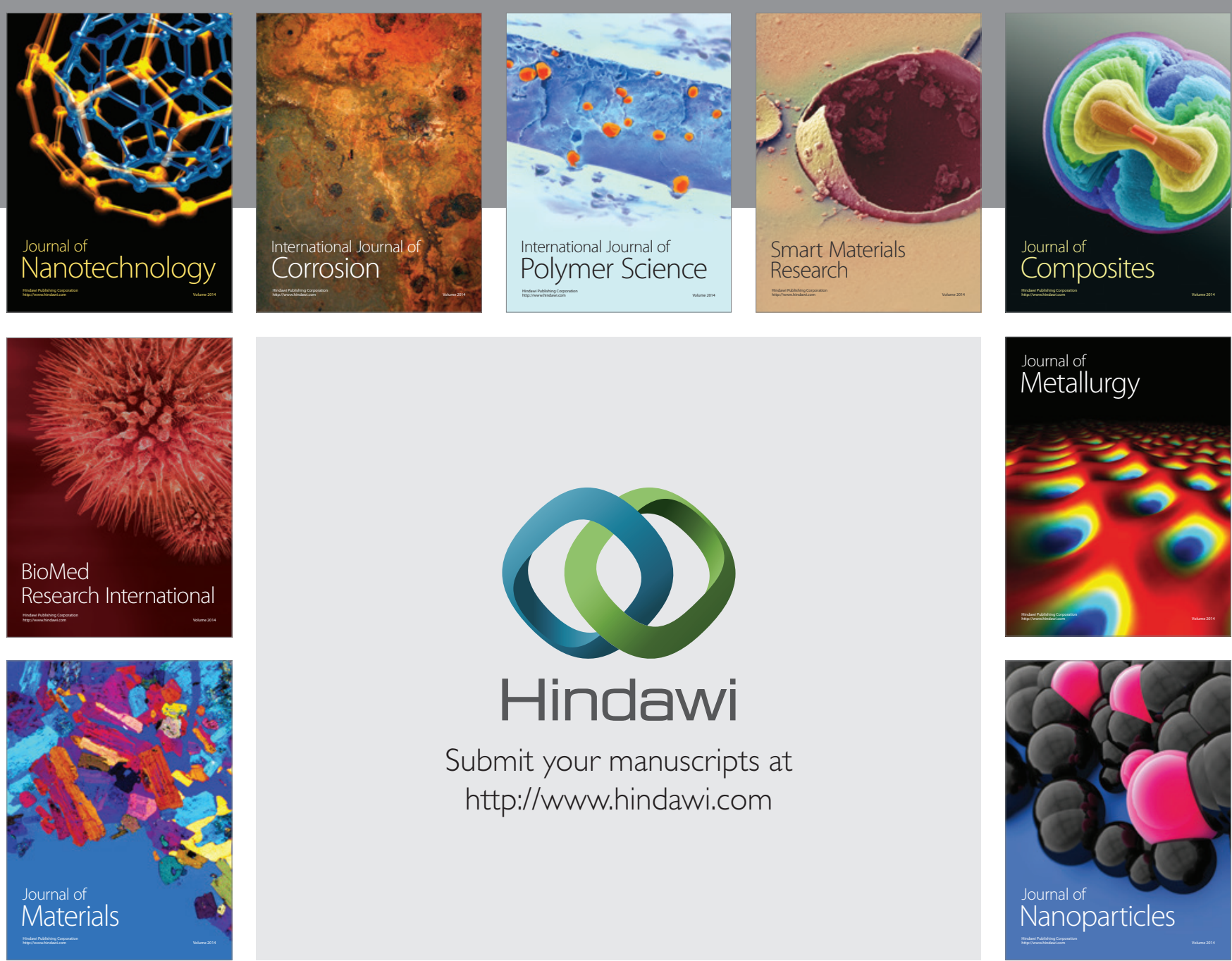

\section{Hindawi}

Submit your manuscripts at

http://www.hindawi.com

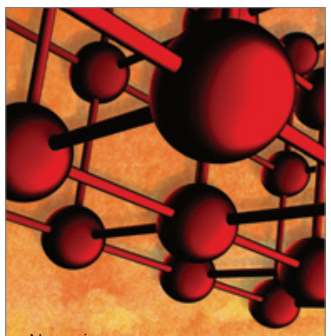

Materials Science and Engineering
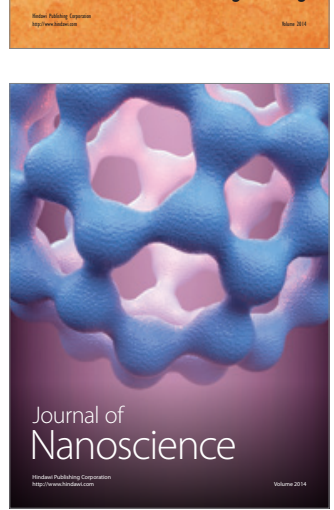
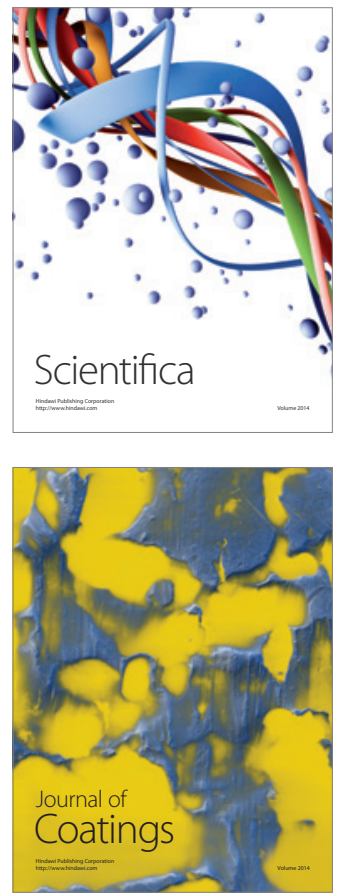
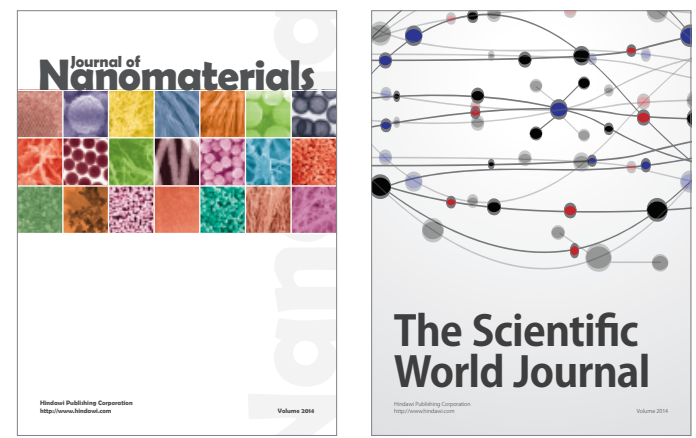

The Scientific World Journal
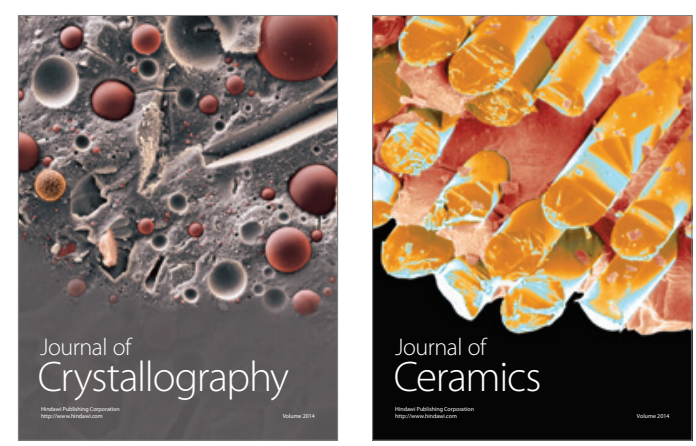
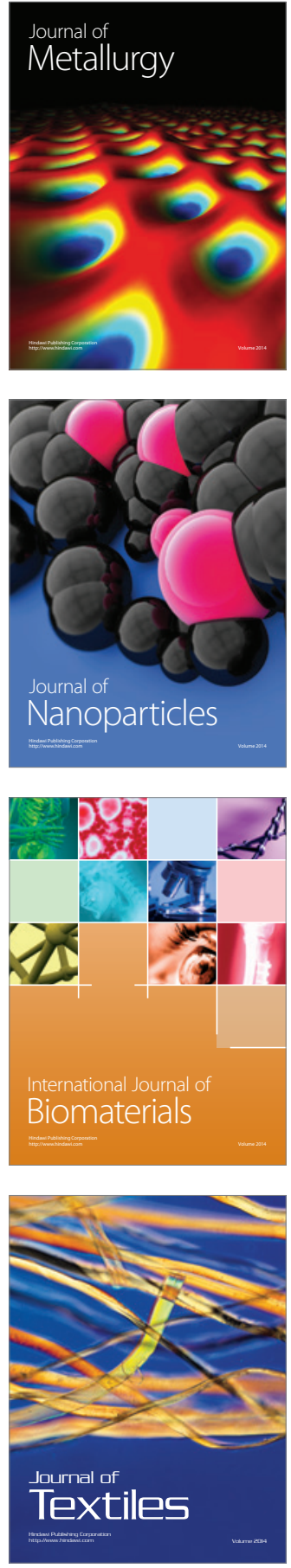